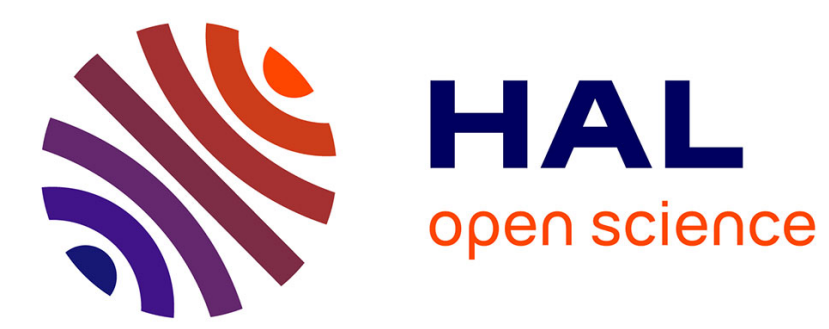

\title{
On the stability of a triplet of scoring rules
}

Mostapha Diss, Vincent Merlin

\section{To cite this version:}

Mostapha Diss, Vincent Merlin. On the stability of a triplet of scoring rules. Theory and Decision, 2010, 69, pp.289-316. 10.1007/s11238-009-9187-6 . halshs-00443854

\section{HAL Id: halshs-00443854 https://shs.hal.science/halshs-00443854}

Submitted on 4 Jan 2010

HAL is a multi-disciplinary open access archive for the deposit and dissemination of scientific research documents, whether they are published or not. The documents may come from teaching and research institutions in France or abroad, or from public or private research centers.
L'archive ouverte pluridisciplinaire HAL, est destinée au dépôt et à la diffusion de documents scientifiques de niveau recherche, publiés ou non, émanant des établissements d'enseignement et de recherche français ou étrangers, des laboratoires publics ou privés. 


\title{
ON THE STABILITY OF A TRIPLET OF SCORING RULES *
}

\author{
Mostapha Diss ${ }^{\dagger}$ and Vincent Merlin ${ }^{\ddagger}$ \\ Preliminary version, January 2008
}

\begin{abstract}
When choosing a voting rule in order to make subsequent decisions, the members of a committee may wish this rule to be self-selected when it is the object of a choice among a menu of different possible voting rules. Such concepts have recently been explored in Social Choice theory, and a menu of voting rule is said to be stable if it contains at least one self-selective voting rule at each profile of preferences on voting rules. We consider in this paper the menu constituted by the three well-known scoring rules \{Borda, Plurality, Antiplurality\}. Under the Impartial Cultures Assumption (IC), which proposes an a priori model to estimate the likelihood of the profiles, we will derive a probability for the stability of this triplet of voting rules.
\end{abstract}

KEY WORDS: Self-selectivity, Stability, Instability, Scoring Rules, Impartial Culture Assumption.

\section{Introduction}

Since long, Social Choice theory has tried to study and model the construction of collective choices upon individual choices. The applications of this theory are widely spread since the actual options may be social states, political candidates, investments, etc. But the choice always concerns alternatives (political candidates for example) and, until recently, little attention has been paid to the choice of the voting rule itself.

Koray (2000), Koray and Unel (2003), Barberà and Jackson (2004), Barberà and Beviá (2002) and Houy (2005) have been the first to tackle that question and enrich, thereby, the traditional field of the Social Choice Theory. The individuals are thus able to choose how to choose and compare the voting rules on the basis of

\footnotetext{
*We gratefully acknowledge Nicolas Houy for helpful comments and suggestions.

†CREM, UMR6211, Université de Caen, Faculté de sciences économiques et de gestion, 19 rue Claude Bloch, 14000 Caen, France. Email: mostapha.diss@unicaen.fr

${ }^{\ddagger}$ CREM, UMR6211, CNRS and Université de Caen, Faculté de sciences économiques et de gestion, 19 rue Claude Bloch, 14000 Caen, France. Email: vincent.merlin@unicaen.fr
} 
their properties. These authors show how that enrichment allows to introduce new positive axioms and new possibility and impossibility results.

One of the new principle introduced by this literature is the notion of selfselectivity. Consider a set of possible voting rules. A voting rule is self-selective (or self stable) if there is no alternative rule that beat the given voting rule if the given voting rule is used to choose between the rules. Let us try to illustrate this concept through some examples. Imagine that the votes are made according to the majority rule: when there is a majority favorable to the majority voting rule, the majority decision is self-selective. On the other hand, if the dictator is favorable to the majority voting rule, dictatorship is not self-selective. This exactly what happened in Spain: the first decision imposed by the King Juan Carlos was to get rid of his dictatorship in order to establish a democracy. The issue is then to know whether self-selective voting rules exist.

Though Barberà and Jackson have applied these ideas to the set of quota rules, until now, no paper has tried to apply this concept to a set of famous voting rules, the scoring voting rules. The objective of this paper is to consider the triplet that forms the three most well known scoring rules: the Borda method (B), the Plurality rule (P) and the Antiplurality rule (A). In this paper, we will also depart from the consequentialism hypothesis which have been assumed by most of the authors, Nicolas Houy being sole exception. Thus, individual will judge and evaluate the scoring rules on the basis of their intrinsic properties, without taking into account the fact that the choice of a voting rule may be instrumental for a voter, in order to increase the chances of the alternatives he prefers.

Though the previous examples have illustrated the notion of self-selectivity for a given voting rule, the concept becomes more subtle when it comes to judge the stability of a menu of possible decision rules. We will consider the definition proposed by Houy (2005): A set of voting rules is (weakly) stable if there always exists at least one self-selective rule at any profile. Notice however that stronger definitions can be adopted, taking into account the fact that the dynamic of designations should always drive the results to a self-selective voting rules (for more details on the different concepts of stability, see Houy (2005)).

Even with this definition of stability, it is not sure whether any set of rules is stable: In fact, Nicolas Houy proposed necessary and sufficient conditions for the stability of a set of neutral ${ }^{1}$ rules. However, the conditions he proposed are very general, and he did not seek to apply his concepts to a set of "classical" rules, like the scoring rules, which are not only neutral, but also anonymous ${ }^{2}$ We will first show that the triplet $\{$ Borda, Plurality, Antiplurality $\}$ may not be stable at some preference profile. Next, we will evaluate the probability that the triplet

\footnotetext{
${ }^{1}$ Following May (1952), a voting rule is neutral is any permutation on the names of alternatives also permutes the social result accordingly. It guaranties that no alternative is favored by the voting rule.

${ }^{2}$ Anonymity has also been defined by May (1952). A rule is anonymous if the social result is unaffected by permutations on the names of the voters. This condition ensures that all the voters are treated equally.
} 
\{Borda, Plurality, Antiplurlity $\}$ is stable in a very weak sense: at each profile, there should at least one self-selective rule.

The likelihood of stability of this voting triplet necessarily depends upon the hypothesis that are set upon the behavior of the voters. We will assume that each individual have the same probability to pick any of the possible preference types. This hypothesis is known as the Impartial Culture model (IC) in Social Choice literature. We will outline a more concise definition of that hypothesis in section 2 .

Regarding the technical aspects, we will use a method based upon the Schläfli's formula in order to derive the desired probabilities. This technique has been widely used in various articles these last years. See Saari and Tataru (1999), Tataru and Merlin (1997) and Merlin and al. (2000, 2002).

The paper will be organized as follows: After reminding some basic definitions dealing with preferences, scoring rules, and probability models in Section 2, we will go through the different aspects of the stability of the \{Borda, Plurality, Antiplurality triplet and the different cases in Section 3. Section 4 is devoted to the computations. Finally section 5 presents our conclusions.

\section{Notations and Definitions}

\subsection{The Preferences}

Throughout all the paper, we will suppose that every voter can rank the set of candidates (here, voting rules) without ties. Preferences will then be linear orders. We will denote by $a b c$ the fact that candidate $a$ is preferred to candidate $b$ who is himself preferred to candidate $c$. In addition to that we will suppose that the voters are honest, i.e. they reveal their true preferences.

We will assume that the set $X=\{$ Borda, Plurality, Antiplurality $\}$ is the menu proposed to the $n$ individuals. The $3 !=6$ types (with strict and transitive preferences) of preferences are displayed in Table 1 and enumerated from 1 to $6, n_{i}$ being the number of agents having the preference type number $i$ :

Table 1: The six preference types among $\{B, P, A\}$

$$
A P B\left(n_{1}\right) \quad A B P\left(n_{2}\right) \quad B A P\left(n_{3}\right) \quad B P A\left(n_{4}\right) \quad P B A\left(n_{5}\right) \quad P A B\left(n_{6}\right)
$$

The existence or not, of a self-selective rule will thus be determined by the study of the situation represented by the vector $\tilde{n}=\left(n_{1}, \ldots, n_{6}\right)$ which describes the

number of electors according to their preferences, with $\sum_{i=1}^{6} n_{i}=n$. The set of possible profiles for a size $n$ population is then:

$$
\mathcal{D}^{n}=\left\{\tilde{n}=\left(n_{1}, \ldots, n_{6}\right), \sum_{i=1}^{6} n_{i}=n\right\}
$$


As we may consider variable electorates, we will define the voting rules on:

$$
\mathcal{D}=\cup_{n=1}^{\infty} \mathcal{D}^{n}
$$

\section{$2.2 \quad$ Scoring Rules}

A voting rule will is thus a mapping from $\mathcal{D}$ into $2^{X} \backslash \emptyset$. At this stage, we assume that a voting rule is a social choice correspondence, that is, it associate to each profile at least one (winning) alternative. A scoring voting rule is a social choice correspondence which depends upon the positions the alternatives occupy in the preference orderings of the individuals taking part in the collective decision. In other terms, each individual gives $P_{1}$ points to the candidate, whom he considers first, $P_{2}$ to the next, until $P_{m}$ points for the last candidate, providing that $P_{1} \geq P_{2} \geq \ldots \geq P_{m}$ and $P_{1}>P_{m}$. The candidate who has received the greatest amount of points over the whole population is elected. Our work will only be dealing with the case where three options are considered, i.e. $m=3$.

Hence, for any $-1 \leq s \leq 1$, let us consider the following family of point vectors $W_{s}=(2,1+s, 0)$; This family describes all the possible scoring rules for the case of 3 options. In particular, $W_{s}$ is the Plurality rule for $s=-1$, the Borda rule for $s=0$ and the Antiplurality rule if $s=1$. In fact, We will study the stability of the triplet $\left\{B, W_{-s}, W_{s}\right\}$, that will allow us to get easily results for the triplet $\{$ Borda, Plurality, Antiplurality $\}$ for the case $s=1$.

With the preferences described in Table 1 and a vector $\tilde{n}=\left(n_{1}, n_{2}, n_{3}, n_{4}, n_{5}, n_{6}\right)$, the application of the Borda rule, defined by the vector $W_{s}=(2,1,0)$, on the triplet \{Borda, Plurality, Antiplurality\} gives us the following scores:

$$
\left\{\begin{array}{l}
S\left(W_{0}, \tilde{n}, A\right)=2\left(n_{1}+n_{2}\right)+\left(n_{3}+n_{6}\right) \\
S\left(W_{0}, \tilde{n}, B\right)=2\left(n_{3}+n_{4}\right)+\left(n_{2}+n_{5}\right) \\
S\left(W_{0}, \tilde{n}, P\right)=2\left(n_{5}+n_{6}\right)+\left(n_{1}+n_{4}\right)
\end{array}\right.
$$

with $S\left(W_{0}, \tilde{n}, A\right)$ being the score of $A$ obtained from the application of the rule $s=0$ to the vector $\tilde{n}=\left(n_{1}, n_{2}, n_{3}, n_{4}, n_{5}, n_{6}\right)$. More generally, the application of the rule defined by $W_{s}=(2,1+s, 0)$ to the same triplet allows us to find the following scores:

$$
\left\{\begin{array}{l}
S\left(W_{s}, \tilde{n}, A\right)=2\left(n_{1}+n_{2}\right)+(1+s)\left(n_{3}+n_{6}\right) \\
S\left(W_{s}, \tilde{n}, B\right)=2\left(n_{3}+n_{4}\right)+(1+s)\left(n_{2}+n_{5}\right) \\
S\left(W_{s}, \tilde{n}, P\right)=2\left(n_{5}+n_{6}\right)+(1+s)\left(n_{1}+n_{4}\right)
\end{array}\right.
$$

We thus find the antiplurality case for $s=1$.

The application of the rule given by $W_{-s}=(2,1-s, 0)$ provides the following expressions for the scores:

$$
\left\{\begin{array}{l}
S\left(W_{-s}, \tilde{n}, A\right)=2\left(n_{1}+n_{2}\right)+(1-s)\left(n_{3}+n_{6}\right) \\
S\left(W_{-s}, \tilde{n}, B\right)=2\left(n_{3}+n_{4}\right)+(1-s)\left(n_{2}+n_{5}\right) \\
S\left(W_{-s}, \tilde{n}, P\right)=2\left(n_{5}+n_{6}\right)+(1-s)\left(n_{1}+n_{4}\right)
\end{array}\right.
$$

We thus find, the plurality case when $s=1$. 


\subsection{The Impartial Culture Assumption}

The Impartial Culture hypothesis (IC) is one of the most used hypothesis in the Social Choice literature in order to describe a priori the likelihood of each particular profile. This hypothesis has been introduced for the first time in literature for the social choice by Guilbauld (1952) in order to study the Condorcet paradox. The basic idea of the Impartial Culture $I C$ model is that each voter selects randomly and independently his preferences from a uniform distribution on the set of possible types. Hence, all the profiles have the same apparition probability and the occurrence of a voting situation $\tilde{n}=\left(n_{1}, \ldots, n_{m !}\right)$ is given by:

$$
\operatorname{Pr}(\tilde{n})=\frac{n !}{n_{1} ! n_{2} ! \ldots n_{m !} !}\left(\frac{1}{m !}\right)^{n}
$$

In the case of 3 alternatives, each voter picks any of the preference types displayed

on Table 1 with probability $\frac{1}{6}$; we obtain $\operatorname{Pr}(\tilde{n})=\frac{n !}{n_{1} ! n_{2} ! \ldots n_{6} !}(6)^{-n}$. A reader who wants to improve his knowledge about the use of probability assumptions in voting theory may consult Berg and Lepelley (1994) or Gehrlein (2002, 2006).

\section{The Stability of \{Borda, Plurality, Antiplurality}

\subsection{A Definition for Stability}

We will now present the definition of stability which we will then apply to the triplet \{Borda, Plurality, Antiplurality\}. Under the hypothesis of a universal domain, if one proposes to a society a set of neutral and different voting rules, could we be sure that the preferences will lead to a self-selective rule chosen by the individuals? In a global aspect, the notion of stability concerns the choice of one rule among many of them, independent of initial preferences. Moreover, on applying this notion, one rule may be considered as preferred to another if it is more stable within a given initial menu of voting rules.

Definition 1 Consider a set of voting rules $E$, and a profile of linear preferences on these voting rules. A voting rule is self-selective at some profile if the choice of the voting rule is this given rule for this profile. The set $E$ is weakly stable if, at any profile, there exists at least one self-selective voting rule.

Let us consider two examples to highlight these ideas. We will first consider a society of 6 individuals. We propose to that society the menu of rules $E=\{$ Borda, Plurality, Antiplurality $\}$. The individual preferences are displayed in Table 2. For this example, only the Borda rule is self-selective because the application of Borda gives out Borda as a result (9 points for $\mathrm{B}, 5$ for $\mathrm{P}$ and 4 for $\mathrm{A}$ ). On the other hand, the application of the Plurality and Antiplurality gives out Borda as a result.

However, Table 3 shows that the menu $\{B, P, A\}$ may not be stable. With the preferences displayed in Table 3, we observe that the Antiplurality rule selects the 
Table 2: An example for stability

$$
B P A(2) \quad B A P(2) \quad P B A(1) \quad A P B(1)
$$

Table 3: An example of instability

$$
B P A(1) \quad P B A(2) \quad A P B(4)
$$

Plurality rule (14 points for P, 8 for A and 6 for B), the Borda count picks out the Plurality rule (9 points for P, 8 for A and 4 for B) which itself leads to the choice of the Antiplurality rule ( 8 points for A, 4 for $\mathrm{P}$ and 2 for $\mathrm{B}$ ). Thus, our objective is to try to calculate the stability probability of this set of 3 voting rules under the Impartial Culture Hypothesis. To fulfil this programme, we must first enumerate all the possible relationships among the three scoring rules.

\subsection{The Different Cases}

In this study, we will first assume that the size of the population is large, which will make the probabilities of tied outcomes extremely unlikely ${ }^{3}$. Thus, the application of each rule to the triplet $\{$ Borda, Plurality, Antiplurality\} only gives the three possible singletons $\{$ Borda $\},\{$ Plurality $\}$ or $\{$ Antiplurality $\}$ as a result. In fact, taking aside the unlikely cases where the results is a tie, we will obtain $(3)^{3}=27$ possible cases. We can partition the 27 cases into 6 categories, represented in the Table 4.

Table 4: The six possible categories

\begin{tabular}{||c|c|c||}
\hline Categories & Cycles & Number of self-selective rule \\
\hline I & Cycle with 3 & 0 \\
II & Cycle with 2 & 0 \\
III & Cycle with 2 & 1 \\
IV & 0 cycle & 1 \\
V & 0 cycle & 2 \\
VI & 0 cycle & 3 \\
\hline
\end{tabular}

All the 27 cases then fall into one of the six categories (see Table 5). The example displayed on Table 2 corresponds to case 17, and the cycle of Table 3 corresponds to case 2.

\footnotetext{
${ }^{3}$ Marchant (2001) studied the probability of apparition of each tie for scoring voting rules (Borda, Plurality...). He points out some analytical and numerical results, proving that this probability tends towards zero when the number of electors is very large.
} 
Table 5: All the possible cases

\begin{tabular}{||c|c|c|c||}
\hline \multicolumn{1}{|c|}{} & \multicolumn{3}{|c||}{ the voting rule } \\
\hline Case $N^{0}$ & $B$ & $P$ & $A$ \\
\hline 1 & $\mathrm{~A}$ & $\mathrm{~B}$ & $\mathrm{P}$ \\
2 & $\mathrm{P}$ & $\mathrm{A}$ & $\mathrm{B}$ \\
\hline
\end{tabular}

Table 5.a : Category I

\begin{tabular}{||c|c|c|c||}
\hline \multicolumn{1}{|c|}{} & \multicolumn{3}{|c||}{ the voting rule } \\
\hline Case $N^{0}$ & $B$ & $P$ & $A$ \\
\hline 9 & $\mathrm{P}$ & $\mathrm{B}$ & $\mathrm{A}$ \\
10 & $\mathrm{~B}$ & $\mathrm{~A}$ & $\mathrm{P}$ \\
11 & $\mathrm{~A}$ & $\mathrm{P}$ & $\mathrm{B}$ \\
\hline
\end{tabular}

Table 5.c : Category III

\begin{tabular}{||c|c|c|c||}
\hline & \multicolumn{3}{|c||}{ the voting rule } \\
\hline Case $N^{0}$ & $B$ & $P$ & $A$ \\
\hline 21 & $\mathrm{~B}$ & $\mathrm{~B}$ & $\mathrm{~A}$ \\
22 & $\mathrm{~B}$ & $\mathrm{~A}$ & $\mathrm{~A}$ \\
23 & $\mathrm{~A}$ & $\mathrm{P}$ & $\mathrm{A}$ \\
24 & $\mathrm{P}$ & $\mathrm{P}$ & $\mathrm{A}$ \\
25 & $\mathrm{~B}$ & $\mathrm{P}$ & $\mathrm{B}$ \\
26 & $\mathrm{~B}$ & $\mathrm{P}$ & $\mathrm{P}$ \\
\hline
\end{tabular}

Table 5.e : Category V

\begin{tabular}{||c|c|c|c||}
\hline \multicolumn{1}{|c|}{} & \multicolumn{3}{|c||}{ the voting rule } \\
\hline Case $N^{0}$ & $B$ & $P$ & $A$ \\
\hline 3 & $\mathrm{~A}$ & $\mathrm{~A}$ & $\mathrm{P}$ \\
4 & $\mathrm{~A}$ & $\mathrm{~A}$ & $\mathrm{~B}$ \\
5 & $\mathrm{P}$ & $\mathrm{B}$ & $\mathrm{B}$ \\
6 & $\mathrm{~A}$ & $\mathrm{~B}$ & $\mathrm{~B}$ \\
7 & $\mathrm{P}$ & $\mathrm{B}$ & $\mathrm{P}$ \\
8 & $\mathrm{P}$ & $\mathrm{A}$ & $\mathrm{P}$ \\
\hline
\end{tabular}

Table 5.b : Category II

\begin{tabular}{||c|c|c|c||}
\hline \multicolumn{1}{|c|}{} & \multicolumn{3}{|c||}{ the voting rule } \\
\hline Case $N^{0}$ & $B$ & $P$ & $A$ \\
\hline 12 & $\mathrm{~A}$ & $\mathrm{~B}$ & $\mathrm{~A}$ \\
13 & $\mathrm{P}$ & $\mathrm{A}$ & $\mathrm{A}$ \\
14 & $\mathrm{~A}$ & $\mathrm{~A}$ & $\mathrm{~A}$ \\
15 & $\mathrm{~B}$ & $\mathrm{~A}$ & $\mathrm{~B}$ \\
16 & $\mathrm{~B}$ & $\mathrm{~B}$ & $\mathrm{P}$ \\
17 & $\mathrm{~B}$ & $\mathrm{~B}$ & $\mathrm{~B}$ \\
18 & $\mathrm{~A}$ & $\mathrm{P}$ & $\mathrm{P}$ \\
19 & $\mathrm{P}$ & $\mathrm{P}$ & $\mathrm{B}$ \\
20 & $\mathrm{P}$ & $\mathrm{P}$ & $\mathrm{P}$ \\
\hline
\end{tabular}

Table 5.d : Category IV

\begin{tabular}{||c|c|c|c||}
\hline & \multicolumn{3}{|c||}{ the voting rule } \\
\hline Case $N^{0}$ & $B$ & $P$ & $A$ \\
\hline 27 & $\mathrm{~B}$ & $\mathrm{P}$ & $\mathrm{A}$ \\
\hline
\end{tabular}

Table 5.f : Category VI

\subsection{Case 1 in details}

Let us consider, for example, the inequalities that characterize the first case presented on Table 5. Since B gives A, A gives P, and P gives B, we will study the system (5), and let $s$ go to 1 . 


$$
\begin{cases}I_{1}: & S\left(W_{0}, \tilde{n}, A\right)>S\left(W_{0}, \tilde{n}, P\right) \\ I_{2}: & S\left(W_{0}, \tilde{n}, A\right)>S\left(W_{O} \tilde{n}, B\right) \\ I_{3}: & S\left(W_{-s}, \tilde{n}, B\right)>S\left(W_{-s}, \tilde{n}, P\right) \\ I_{4}: & S\left(W_{-s}, \tilde{n}, B\right)>S\left(W_{-s}, \tilde{n}, A\right) \\ I_{5}: & S\left(W_{s}, \tilde{n}, P\right)>S\left(W_{s}, \tilde{n}, B\right) \\ I_{6}: & S\left(W_{s}, \tilde{n}, P\right)>S\left(W_{s}, \tilde{n}, A\right)\end{cases}
$$

This set of inequalities is equivalent to:

$$
\begin{cases}I_{1}: & n_{1}+2 n_{2}+n_{3}-n_{4}-2 n_{5}-n_{6}>0 \\ I_{2}: & 2 n_{1}+n_{2}-n_{3}-2 n_{4}-n_{5}+n_{6}>0 \\ I_{3}: & (-1+s) n_{1}+(1-s) n_{2}+2 n_{3}+(1+s) n_{4}+(-1-s) n_{5}-2 n_{6}>0 \\ I_{4}: & -2 n_{1}+(-1-s) n_{2}+(1+s) n_{3}+2 n_{4}+(1-s) n_{5}+(-1+s) n_{6}>0 \\ I_{5}: & (1+s) n_{1}+(-1-s) n_{2}-2 n_{3}+(-1+s) n_{4}+(1-s) n_{5}+2 n_{6}>0 \\ I_{6}: & (-1+s) n_{1}-2 n_{2}+(-1-s) n_{3}+(1+s) n_{4}+2 n_{5}+(1-s) n_{6}>0\end{cases}
$$

In the same way, we can easily write down the different inequalities for the other 26 cases. In order to limit the efforts and make our computations easier, we may state several remarks ${ }^{2}$. With the help of elementary mathematical operations, we can easily demonstrate that 6 cases are impossible $\left(5^{\text {th }}, 6^{\text {th }}, 13^{\text {th }}, 18^{\text {th }}, 22^{\text {th }}\right.$ et $26^{\text {th }}$ case). The $5^{t h}$ case is discussed as an example in Appendix I. In order to facilitate the resolution, notice that many cases can be re-written in the form of reduced systems, with 4 inequalities only instead of 6 . Let us consider the first case being defined by the system (6). Easy computation demonstrate that $I_{4}+I_{6}+2 I_{1}=I_{3}$ and $I_{4}+I_{6}+2 I_{2}=I_{5}$. We can thus reduce our system of inequalities into (7):

$$
\begin{cases}I_{1}: & n_{1}+2 n_{2}+n_{3}-n_{4}-2 n_{5}-n_{6}>0 \\ I_{2}: & 2 n_{1}+n_{2}-n_{3}-2 n_{4}-n_{5}+n_{6}>0 \\ I_{4}: & -2 n_{1}+(-1-s) n_{2}+(1+s) n_{3}+2 n_{4}+(1-s) n_{5}+(-1+s) n_{6}>0 \\ I_{6}: & (-1+s) n_{1}-2 n_{2}+(-1-s) n_{3}+(1+s) n_{4}+2 n_{5}+(1-s) n_{6}>0\end{cases}
$$

We will present in Appendix II the other reduced systems $\left(1^{s t}, 2^{\text {nd }}, 9^{\text {th }}, 10^{\text {th }}, 11^{\text {th }}\right.$, $14^{\text {th }}, 17^{\text {th }}, 20^{\text {th }}$, and the $27^{\text {th }}$ case). For the remaining cases, we will use an indirect method. In order to calculate the probability of the $3^{\text {rd }}$ case, we will use the results obtained for the $1^{\text {st }}$ and the $18^{\text {th }}$ cases. Denote by $\operatorname{Pr}(X)$ the probability of an event $X$. Thus:

$$
\operatorname{Pr}(\text { case } 1)=\operatorname{Pr}(A \rightarrow P, B \rightarrow A, P \rightarrow B)
$$

$A \rightarrow P$ means that the application of the Antiplurality rule to the triplet $\{B, P, A\}$ gives out the Plurality as a results. We may thus write that:

$$
\operatorname{Pr}(A \rightarrow P \text { et } B \rightarrow A)=\operatorname{Pr}(\text { case } 1)+\operatorname{Pr}(\text { case } 3)+\operatorname{Pr}(\text { case } 18)
$$


We will see further on that we will be able to calculate $\operatorname{Pr}(A \rightarrow P$ et $B \rightarrow A)$. But we already know that $\operatorname{Pr}($ case 18$)=0$. We can finally write that:

$$
\operatorname{Pr}(\text { case } 3)=\operatorname{Pr}(A \rightarrow P \text { et } B \rightarrow A)-\operatorname{Pr}(\text { case } 1)
$$

We will be using the same method in order to simplify the calculus of the probability of each remaining cases $\left(4^{t h}, 7^{t h}, 8^{t h}, 12^{t h}, 15^{t h}, 16^{t h}, 19^{t h}, 21^{t h}, 23^{t h}, 24^{t h}, 25^{t h}\right)$. The details for all the cases are given in Appendix III. In fact, we will need to calculate the likelihood of the cases displayed in Table 6 where, each time, 4 inequalities are sufficient.

Table 6: The cases to consider for probability computations

$$
\begin{aligned}
\operatorname{Pr}(A \rightarrow P \text { and } B \rightarrow A) & \operatorname{Pr}(A \rightarrow P \text { and } B \rightarrow B) \\
\operatorname{Pr}(A \leftrightarrows B) & \operatorname{Pr}(A \rightarrow B \text { and } B \rightarrow P) \\
\operatorname{Pr}(P \leftrightarrows B) & \operatorname{Pr}(A \rightarrow A \text { and } B \rightarrow B) \\
\operatorname{Pr}(A \rightarrow P \text { and } B \rightarrow P) & \operatorname{Pr}(A \rightarrow A \text { and } B \rightarrow A) \\
\operatorname{Pr}(P \rightarrow B \text { and } B \rightarrow A) & \operatorname{Pr}(A \rightarrow A \text { and } B \rightarrow P) \\
\operatorname{Pr}(P \rightarrow A \text { and } B \rightarrow B) & \operatorname{Pr}(A \rightarrow B \text { and } B \rightarrow B)
\end{aligned}
$$

\section{Results : probabilities computations}

\subsection{Case 1 in details}

The method used in order to compute the probability of voting events has been developed by Saari and Tataru (1999), and further developed by Merlin, Tataru and Valognes (2000, 2002) and Merlin and Valognes (2004). Under IC assumption, each voter is equally likely to pick independently any of the six preference types. By using Central Limit Theorem which implies, when $n$ tends towards infinity, the following formula in $\mathbb{R}$ holds for $n_{i}$ :

$$
\operatorname{Pr}\left(a<\frac{\left(n_{i}-\frac{n}{6}\right)}{\sigma \sqrt{n}}<b\right) \mapsto \frac{1}{\sqrt{2} \pi} \int_{a}^{b}\left(e^{\frac{-t^{2}}{2}} d t\right), \quad n \rightarrow \infty
$$

The measure of $\frac{\left(n_{i}-\frac{n}{6}\right)}{\sigma \sqrt{n}}$ noted $\mu\left(\frac{n_{i}-\frac{n}{6}}{\sigma \sqrt{n}}\right)$ verify the following relation in $\mathbb{R}: \mu\left(\frac{n_{i}-\frac{n}{6}}{\sigma \sqrt{n}}\right) \mapsto$ $\frac{1}{\sqrt{2} \pi} e^{\frac{-t^{2}}{2}} \lambda$, where $\lambda$ is the Lebesgue measure on the one dimensional hyperplane. In addition, the resolution of our systems requires an application of this theorem in $\mathbb{R}^{5}$ : 
Let

$$
\mathbf{m}=\left(m_{1}, m_{2}, \ldots m_{6}\right)^{T}=\frac{\left(\begin{array}{c}
n_{1} \\
\vdots \\
n_{6}
\end{array}\right)-\left(\begin{array}{c}
\frac{n}{6} \\
\vdots \\
\frac{n}{6}
\end{array}\right)}{\sigma \sqrt{n}}
$$

The Central Limit Theorem in $\mathbb{R}^{5}$ implies, when $n$ tends towards infinity, that :

$$
\mu(\mathbf{m}) \mapsto \frac{1}{(\sqrt{2} \pi)^{5}} e^{\frac{-|t|^{2}}{2}} \lambda
$$

where $t=\left(t_{1}, t_{2}, \ldots, t_{6}\right) \in \mathbb{R}^{6} ;|t|^{2}=t_{1}^{2}+\ldots+t_{6}^{2}$ and $\lambda$ is the Lebesgue measure on the five dimensional hyperplane $\left(t_{1}+\ldots+t_{6}=0\right)$. As stated above, the fact that the Antiplurality designates Plurality, that Borda designates Antiplurality and that Plurality designates Borda is characterized by the four inequalities of the system (7):

To compute the probability of this voting situation we will use the following reasoning. $\mathbf{m}$ verify the inequalities of the system $(7)$ if and only if $\tilde{n}=\left(n_{1} \ldots n_{6}\right)$ verify them too. Then we write :

$$
\operatorname{Pr}(\mathbf{m} \text { verifies }(7)) \mapsto \frac{1}{(\sqrt{2} \pi)^{5}} \int_{C_{1}}\left(e^{\frac{-|t|^{2}}{2}}\right) d \lambda
$$

where

$$
C_{1}=\left\{\mathbf{m} \in \mathbb{R}^{5}, \mathbf{m} \text { verifies }(7), \sum_{j=1}^{6} \mathbf{m}_{j}=0\right\}
$$

The measure $\bar{\mu} \equiv \frac{1}{(\sqrt{2} \pi)^{5}} e^{\frac{-|t|^{2}}{2}} \lambda$ is absolutely continuous and radially symmetric. Hence, computing $P(\mathbf{m}$ satisfies (7)) reduces to finding the measure of the cone $C_{1}$. That will be done using the Schläfli's formula (See Kellerhals (1989) and Milnor (1982)). This formula gives the differential volume on the set of spherical $n$ simplexes:

$$
\operatorname{dvol}_{n}\left(C_{1}\right)=\frac{1}{(n-1)} \sum_{1 \leq j<k \leq n} \operatorname{vol}_{n-2}\left(F_{j} \cap F_{k}\right) d \alpha_{j k} \quad ; \quad \operatorname{vol}_{0}=1
$$

where $\alpha_{j k}$ is the dihedral angle formed by the facets $F_{j}$ and $F_{k}$ of $C_{1}$, and $n$ indicates the simplex dimension. Saari and Tataru (1999) were the first to present an application of this formula in voting theory in order to compute the probabilities of some voting events. We present in this work the adaptation of this formula for the probability computation of the stability of $\{$ Borda, Plurality, Antiplurality $\}$ triplet. 
Each facet $F_{i}$ of the cone $C_{1}$ corresponds to one of the inequality $I_{i}$ in equation (7):

$$
\left\{\begin{array}{l}
F_{1}: n_{1}+2 n_{2}+n_{3}-n_{4}-2 n_{5}-n_{6}>0 \\
F_{2}: 2 n_{1}+n_{2}-n_{3}-2 n_{4}-n_{5}+n_{6}>0 \\
F_{3}:-2 n_{1}+(-1-s) n_{2}+(1+s) n_{3}+2 n_{4}+(1-s) n_{5}+(-1+s) n_{6}>0 \\
F_{4}:(-1+s) n_{1}-2 n_{2}+(-1-s) n_{3}+(1+s) n_{4}+2 n_{5}+(1-s) n_{6}>0
\end{array}\right.
$$

Let $\overrightarrow{v_{1}}, \overrightarrow{v_{2}}, \overrightarrow{v_{3}}, \overrightarrow{v_{4}}$ the normal vectors to the hyperplanes $F_{1}, F_{2}, F_{3}, F_{4}$, pointing inside the cone $C_{1}$ :

$$
\left\{\begin{array}{l}
\overrightarrow{v_{1}}=(1,2,1,-1,-2,-1) \\
\overrightarrow{v_{2}}=(2,1,-1,-2,-1,1) \\
\overrightarrow{v_{3}}=(-2,-1-s, 1+s, 2,1-s,-1+s) \\
\overrightarrow{v_{4}}=(-1+s,-2,-1-s, 1+s, 2,1-s)
\end{array}\right.
$$

The relation : $\overrightarrow{v_{i}} \cdot \overrightarrow{v_{j}}=\left\|\overrightarrow{v_{i}}\right\| \cdot\left\|\overrightarrow{v_{j}}\right\| \cdot \cos \left(\pi-\alpha_{i j}\right)=-\left\|\overrightarrow{v_{i}}\right\| \cdot\left\|\overrightarrow{v_{j}}\right\| \cdot \cos \left(\alpha_{i j}\right)$ enables us, in addition, to find the value of the 6 following angles $\alpha_{i j}$ :

$$
\begin{aligned}
& \alpha_{12}=\frac{2 \pi}{3} \\
& \alpha_{13}=\alpha_{24}=\arccos \left(\frac{3}{2 \sqrt{3 s^{2}+9}}\right) \\
& \alpha_{14}=\alpha_{23}=\arccos \left(\frac{3}{\sqrt{3 s^{2}+9}}\right) \\
& \alpha_{34}=\arccos \left(\frac{s^{2}-3}{2\left(s^{2}+3\right)}\right)
\end{aligned}
$$

which implies that:

$$
\begin{aligned}
& d \alpha_{12}=0 \\
& d \alpha_{13}=d \alpha_{24}=\frac{\sqrt{3} s}{\left(3+s^{2}\right) \sqrt{9+4 s^{2}}} \\
& d \alpha_{14}=d \alpha_{23}=\frac{\sqrt{3}}{3+s^{2}} \\
& d \alpha_{34}=-\frac{4 \sqrt{3} s}{\left(3+s^{2}\right) \sqrt{9+10 s^{2}+s^{4}}}
\end{aligned}
$$

The vectors $\overrightarrow{v_{1}}, \overrightarrow{v_{2}}, \overrightarrow{v_{3}}, \overrightarrow{v_{4}}$ are perpendicular to the vectors $\overrightarrow{v_{5}}=(1,1,1,1,1,1)$ and $\overrightarrow{v_{6}}=(1,-1,1,-1,1,-1)$. Then we can calculate the vertex $P_{123}=F_{1} \cap F_{2} \cap F_{3}$ by solving the system:

$$
P_{123}:\left\{\begin{array}{l}
n_{1}+2 n_{2}+n_{3}-n_{4}-2 n_{5}-n_{6}=0 \\
2 n_{1}+n_{2}-n_{3}-2 n_{4}-n_{5}+n_{6}=0 \\
-2 n_{1}+(-1-s) n_{2}+(1+s) n_{3}+2 n_{4}+(1-s) n_{5}+(-1+s) n_{6}=0 \\
(-1+s) n_{1}-2 n_{2}+(-1-s) n_{3}+(1+s) n_{4}+2 n_{5}+(1-s) n_{6}>0 \\
n_{1}+n_{2}+n_{3}+n_{4}+n_{5}+n_{6}=0 \\
n_{1}-n_{2}+n_{3}-n_{4}+n_{5}-n_{6}=0
\end{array}\right.
$$


We derive the following results:

$$
P_{123}:\left\{\begin{array}{l}
n_{1}=2 \\
n_{2}=-1 \\
n_{3}=-1 \\
n_{4}=2 \\
n_{5}=-1 \\
n_{6}=-1
\end{array}\right.
$$

By solving similar linear systems, we obtain the other vertices of the cone $C_{1}$ to be equal to:

$$
P_{124}:\left\{\begin{array}{l}
n_{1}=1 \\
n_{2}=-2 \\
n_{3}=1 \\
n_{4}=1 \\
n_{5}=-2 \\
n_{6}=1
\end{array} \quad P_{134}:\left\{\begin{array}{l}
n_{1}=1+s \\
n_{2}=-2 \\
n_{3}=1-s \\
n_{4}=1-s \\
n_{5}=-2 \\
n_{6}=1+s
\end{array} \quad P_{234}:\left\{\begin{array}{l}
n_{1}=2 \\
n_{2}=-1+s \\
n_{3}=-1+s \\
n_{4}=2 \\
n_{5}=-1-s \\
n_{6}=-1-s
\end{array}\right.\right.\right.
$$

Also we compute:

$$
\begin{gathered}
\operatorname{vol}\left(F_{1}, F_{2}\right)=\left(\widehat{P_{123}, P_{124}}\right)=\frac{1}{3} \pi \\
\operatorname{vol}\left(F_{1}, F_{3}\right)=\operatorname{vol}\left(F_{2}, F_{4}\right)=\left(\widehat{P_{123}, P_{134}}\right)=\arccos \left(\frac{3}{2 \sqrt{9+3 s^{2}}}\right) \\
\operatorname{vol}\left(F_{1}, F_{4}\right)=\operatorname{vol}\left(F_{2}, F_{3}\right)=\left(\widehat{P_{124}, P_{134}}\right)=\arccos \left(\frac{3}{\sqrt{9+3 s^{2}}}\right) \\
\operatorname{vol}\left(F_{3}, F_{4}\right)=\left(\widehat{P_{234}, P_{134}}\right)=\pi-\arccos \left(\frac{-3+s^{2}}{2\left(3+s^{2}\right)}\right)
\end{gathered}
$$

We can now easily apply the Schläfli's formula:

$$
\operatorname{dvol}(C)=2 \operatorname{vol}\left(F_{1}, F_{3}\right) d \alpha_{13}+2 \operatorname{vol}\left(F_{1}, F_{4}\right) d \alpha_{14}+\operatorname{vol}\left(F_{3}, F_{4}\right) d \alpha_{34}
$$

We find:

$$
\begin{gathered}
\frac{d v o l(C)}{d s}=\frac{2 \sqrt{3} s \arccos \left(\frac{3}{2 \sqrt{9+3 s^{2}}}\right)}{\left(3+s^{2}\right) \sqrt{9+4 s^{2}}}+\frac{2 \sqrt{3} \arccos \left(\frac{3}{\sqrt{9+3 s^{2}}}\right)}{3+s^{2}} \\
-\frac{4 \sqrt{3} s\left(\pi-\arccos \left(\frac{-3+s^{2}}{6+2 s^{2}}\right)\right)}{\left(3+s^{2}\right) \sqrt{9+10 s^{2}+s^{4}}}
\end{gathered}
$$


By Dividing the formula by $(n-1)=2$ and $2 \pi^{2}$ (the volume of the unit sphere in $\mathbb{R}^{4}$ ) we obtain:

$$
\begin{aligned}
\operatorname{Pr}(\text { case } 1, s)= & \frac{1}{4 \pi^{2}} \int_{0}^{s}\left(\frac{2 \sqrt{3} t \arccos \left(\frac{3}{2 \sqrt{9+3 t^{2}}}\right)}{\left(3+t^{2}\right) \sqrt{9+4 t^{2}}}+\frac{2 \sqrt{3} \arccos \left(\frac{3}{\sqrt{9+3 t^{2}}}\right)}{3+t^{2}}\right. \\
& \left.-\frac{4 \sqrt{3} t\left(\pi-\arccos \left(\frac{-3+t^{2}}{6+2 t^{2}}\right)\right)}{\left(3+t^{2}\right) \sqrt{9+10 t^{2}+t^{4}}}\right) d t
\end{aligned}
$$

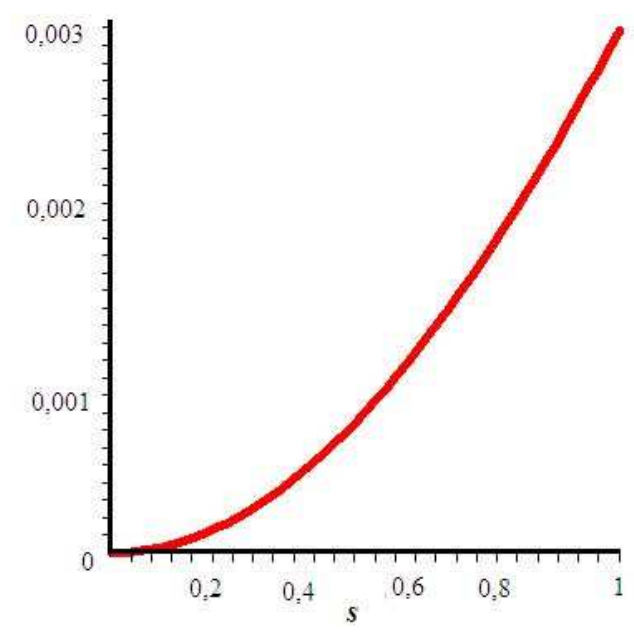

Figure 1: The probability of the $1^{\text {st }}$ case as a function of $\mathrm{s}$

The graphical representation (Figure 1) shows the integration for $0 \leq s \leq 1$. We may find : $\operatorname{Pr}($ case 1$)=\operatorname{Pr}(s=1)=0.00299346$. We will use the same method to find out the other probabilities. We give out all the corresponding probabilities in the Table 7 .

\subsection{General Results}

In fact, when examining carefully the systems, we observe that we only need to obtain three other expressions for the probabilities $\operatorname{Pr}($ cas 1, s) to derive all the other probabilities. Then probabilities for each possible cases are displayed in Table 7. Table 8 sums up the results according to the categories.

We have defined a stable set as being a set which always has a self-selective rule. We may thus state the following proposition:

Proposition 1 : Consider a large population, where each voter has a probability of $1 / 6$ to choose one of the six possible linear orders over the set $E=\{$ Borda, Plurality, Antiplurality\} of voting rules. Then the set $E$ is stable for $84.49 \%$ of the profiles. 
Table 7: The probability for each case

\begin{tabular}{||c|c|c|c||}
\hline Case $\mathrm{N}^{\circ}$ & Probabilities & Case $^{\circ}$ & Probabilities \\
\hline 1 & 0.00299346 & 15 & 0.03728344 \\
2 & 0.00299346 & 16 & 0.03728344 \\
3 & 0.03728344 & 17 & 0.17821261 \\
4 & 0.03728344 & 18 & 0 \\
5 & 0 & 19 & 0.03728344 \\
6 & 0 & 20 & 0.17821261 \\
7 & 0.03728344 & 21 & 0.03728344 \\
8 & 0.03728344 & 22 & 0 \\
9 & 0.00299346 & 23 & 0.03728344 \\
10 & 0.00299346 & 24 & 0.03728344 \\
11 & 0.00299346 & 25 & 0.03728344 \\
12 & 0.03728344 & 26 & 0 \\
13 & 0 & 27 & 0.00299346 \\
14 & 0.17821261 & & \\
\hline
\end{tabular}

Table 8: Probability by category

\begin{tabular}{||c|c|c|c||}
\hline Categories & Cycles & Number of self-selective rule & Probabilities \\
\hline I & Cycle with 3 & 0 & 0.00598692 \\
II & Cycle with 2 & 0 & 0.14913376 \\
\hline III & Cycle with 2 & 1 & 0.00898038 \\
IV & 0 cycle & 1 & 0.68377159 \\
V & 0 cycle & 2 & 0.14913376 \\
VI & 0 cycle & 3 & 0.00299346 \\
\hline
\end{tabular}

We may also learn a few other facts from the results displayed in Tables 7 and 8 . In fact, the instability does not come from cases like example displayed in Table 3. A cycle among the three scoring rules is quite unlikely (less than $0.6 \%$ ). The problem arises when rule 1 designate rule 2 while rule 2 designate rule 1 , the third one leading either to rule 1 and 2.

As a byproduct, we can also compute the probability that all the scoring rules gives the same results. This is the joint probability of cases 14,17 and 20 . We get back a probability of 0.5346 , in accordance with the result previously obtained by Fishburn and Gerhlein with different techniques.

It also possible to use differently the results of Table 7 , in order to examine whether a rule is more likely to be self-selective.

The figures in Table 9 means, for example, that the rule $\{A\}$ (or $\{P\}$ ) is stable 
Table 9: General table

\begin{tabular}{c|c} 
The set & Probabilities \\
\hline$\{A\}$ & $21.85 \%$ \\
$\{B\}$ & $25.58 \%$ \\
$\{P\}$ & $21.85 \%$ \\
\hline$\{A B\}$ & $3.73 \%$ \\
$\{A P\}$ & $7.46 \%$ \\
$\{B P\}$ & $3.73 \%$ \\
\hline$\{A B P\}$ & $0.3 \%$ \\
\hline Instability & $\mathbf{1 5 . 5 1 \%}$
\end{tabular}

alone with $21,85 \%$. $\{A B\}$ are stable alone with $3,73 \%$.

Thus,

$$
\begin{aligned}
\operatorname{Pr}(A \text { is stable alone or with others }) & =\operatorname{Pr}(\{A\})+\operatorname{Pr}(\{A B\}) \\
& +\operatorname{Pr}(\{A P\})+\operatorname{Pr}(\{A B P\}) \\
& =33.33 \%
\end{aligned}
$$

While following the same method we find:

$$
\begin{aligned}
\operatorname{Pr}(B \text { is stable alone or with others }) & =\operatorname{Pr}(P \text { is stable alone or with others }) \\
& =33.33 \% .
\end{aligned}
$$

Therefore, we can say that no rule is more stable than another.

\section{Conclusion}

The objective of this paper was to illustrate the recently growing literature on the self-selectivity of voting rules with a classical example, by considering the three most famous scoring rules. As one may have guessed, the set \{Borda, Plurality, Antiplurality $\}$ is not stable, the problem being mainly concentrated on cycles between two rules.

Thus, is there any escape from impossibility theorems when one consider the choice of a voting rule? Several possibilities could be explored:

- First, we may consider different probability assumptions, considering more homogeneous societies. One possibility would be to consider the Impartial Anonymous Culture, which assumes that all the vectors $\tilde{n}$ are equally likely. This assumption is known to slightly diminish the probability of paradoxical events compared to the IC assumption. Another possibility, quite common in Social Choice theory, is to consider restriction on the preferences, like singledpeaked preferences. 
- In this paper, we have considered rules which seldom give tied outcomes. However, Nicolas Houy has pointed out that one of the key to the stability is the existence of a rule that selects often many rules. So, why not trying such a rule, like for example the Copeland method, which always selects alternatives in the top cycle of the pairwise majority rule? Indeed, for three voters, the set $E=\{$ Borda, Plurality, Copeland $\}$ is stable if we breaks the ties in favor of the statu quo.

- Another possibility would be to consider consequentialism as a way to model the preference of the voters over the voting rules. Consequentialism implies that two voting rules are considered equivalent by the voters if they lead to the selection of the same alternatives. Thus, preferences may now be weak ordering, and it is known the presence of weak orderings in the preference profile tends the reduce the probability of paradoxical outcomes (see Gehrlein and Valognes (2001) and Merlin and Valognes (2002)).

The careful exploration of these possibilities will tell us whether there is any hope of finding reasonably stable menu of voting rules that one can propose with confidence to the choice of the voters.

\section{ACKNOWLEDGEMENTS}

We gratefully thank Nicolas Houy for the comments, remarks and suggestions he made on the different preliminary versions of this paper.

\section{NOTES}

1. We must highlight that several facts can be geometrically demonstrated by using some of Saari's $(1994,1995)$ important results dealing with the geometry of scoring rules. In order to simplify the presentation, these results are not enclosed in the paper. 


\section{Appendix (I)}

The $5^{\text {th }}$ case $(B \rightarrow P, P \rightarrow A, A \rightarrow B)$ is describe by the following system of inequalities:

$$
\begin{cases}I_{1}: & -n_{1}-2 n_{2}-n_{3}+n_{4}+2 n_{5}+n_{6}>0 \\ I_{2}: & n_{1}-n_{2}-2 n_{3}-n_{4}+n_{5}+2 n_{6}>0 \\ I_{3}: & (-1-s) n_{1}+(1+s) n_{2}+2 n_{3}+(1-s) n_{4}+(-1+s) n_{5}-2 n_{6}>0 \\ I_{4}: & -2 n_{1}+(-1+s) n_{2}+(1-s) n_{3}+2 n_{4}+(1+s) n_{5}+(-1-s) n_{6}>0 \\ I_{5}: & -2 n_{1}+(-1-s) n_{2}+(1+s) n_{3}+2 n_{4}+(1-s) n_{5}+(-1+s) n_{6}>0 \\ I_{6}: & (-1+s) n_{1}+(1-s) n_{2}+2 n_{3}+(1+s) n_{4}+(-1-s) n_{5}-2 n_{6}>0\end{cases}
$$

Evaluating $\frac{-1}{2}\left(I_{3}+I_{6}\right)$ enables us to find the inequality

$$
n_{1}-n_{2}-2 n_{3}-n_{4}+n_{5}+2 n_{6}<0
$$

. In comparison with $I_{2}$ we can say that the $5^{\text {th }}$ case is impossible to realize. This result also derives from Saari $(1994,1995)$, which states that in the three candidate case, all the scoring rules coincide if and only if the Plurality rule and the Antiplurality rule select the same winner. Cases $6,13,18,22$ and 26 obey to the same logic.

\section{Appendix (II)}

- $1^{\text {st }}$ case $: B \rightarrow A, \quad P \rightarrow B$ et $A \rightarrow P$

$$
\left\{\begin{array}{l}
n_{1}+2 n_{2}+n_{3}-n_{4}-2 n_{5}-n_{6}>0 \\
2 n_{1}+n_{2}-n_{3}-2 n_{4}-n_{5}+n_{6}>0 \\
-2 n_{1}+(-1+s) n_{2}+(1-s) n_{3}+2 n_{4}+(1+s) n_{5}+(-1-s) n_{6}>0 \\
(-1-s) n_{1}-2 n_{2}+(-1+s) n_{3}+(1-s) n_{4}+2 n_{5}+(1+s) n_{6}>0
\end{array}\right.
$$

- $2^{\text {nd }}$ case $: B \rightarrow P, \quad P \rightarrow A$ et $A \rightarrow B$

$$
\left\{\begin{array}{l}
-n_{1}-2 n_{2}-n_{3}+n_{4}+2 n_{5}+n_{6}>0 \\
n_{1}-n_{2}-2 n_{3}-n_{4}+n_{5}+2 n_{6}>0 \\
(1-s) n_{1}+2 n_{2}+(1+s) n_{3}+(-1-s) n_{4}-2 n_{5}+(-1+s) n_{6}>0 \\
(-1+s) n_{1}+(1-s) n_{2}+2 n_{3}+(1+s) n_{4}+(-1-s) n_{5}-2 n_{6}>0
\end{array}\right.
$$

- $9^{\text {th }}$ case $: B \rightarrow P, \quad P \rightarrow B$ et $A \rightarrow A$

$$
\left\{\begin{array}{l}
-n_{1}-2 n_{2}-n_{3}+n_{4}+2 n_{5}+n_{6}>0 \\
n_{1}-n_{2}-2 n_{3}-n_{4}+n_{5}+2 n_{6}>0 \\
(-1-s) n_{1}+(1+s) n_{2}+2 n_{3}+(1-s) n_{4}+(-1+s) n_{5}-2 n_{6}>0 \\
(1+s) n_{1}+2 n_{2}+(1-s) n_{3}+(-1+s) n_{4}-2 n_{5}+(-1-s) n_{6}>0
\end{array}\right.
$$

- $10^{\text {th }}$ case $: B \rightarrow B, \quad P \rightarrow A$ et $A \rightarrow P$

$$
\left\{\begin{array}{l}
-2 n_{1}-n_{2}+n_{3}+2 n_{4}+n_{5}-n_{6}>0 \\
-n_{1}+n_{2}+2 n_{3}+n_{4}-n_{5}-2 n_{6}>0 \\
2 n_{1}+(1-s) n_{2}+(-1+s) n_{3}-2 n_{4}+(-1-s) n_{5}+(1+s) n_{6}>0 \\
(1-s) n_{1}+(-1+s) n_{2}-2 n_{3}+(-1-s) n_{4}+(1+s) n_{5}+2 n_{6}>0
\end{array}\right.
$$


- $11^{\text {th }}$ case $: B \rightarrow A, \quad P \rightarrow P$ et $A \rightarrow B$

$$
\left\{\begin{array}{l}
n_{1}+2 n_{2}+n_{3}-n_{4}-2 n_{5}-n_{6}>0 \\
2 n_{1}+n_{2}-n_{3}-2 n_{4}-n_{5}+n_{6}>0 \\
(-1+s) n_{1}-2 n_{2}+(-1-s) n_{3}+(1+s) n_{4}+2 n_{5}+(1-s) n_{6}>0 \\
-2 n_{1}+(-1-s) n_{2}+(1+s) n_{3}+2 n_{4}+(1-s) n_{5}+(-1+s) n_{6}>0
\end{array}\right.
$$

- $14^{\text {th }}$ case $: B \rightarrow A, \quad P \rightarrow A$ et $A \rightarrow A$

$$
\left\{\begin{array}{l}
2 n_{1}+(1-s) n_{2}+(-1+s) n_{3}-2 n_{4}+(-1-s) n_{5}+(1+s) n_{6}>0 \\
(1-s) n_{1}+2 n_{2}+(1+s) n_{3}+(-1-s) n_{4}-2 n_{5}+(-1+s) n_{6}>0 \\
2 n_{1}+(1+s) n_{2}+(-1-s) n_{3}-2 n_{4}+(-1+s) n_{5}+(1-s) n_{6}>0 \\
(1+s) n_{1}+2 n_{2}+(1-s) n_{3}+(-1+s) n_{4}-2 n_{5}+(-1-s) n_{6}>0
\end{array}\right.
$$

- $17^{\text {th }}$ case $: B \rightarrow B, \quad P \rightarrow B$ et $A \rightarrow B$

$$
\left\{\begin{array}{l}
(-1-s) n_{1}+(1+s) n_{2}+2 n_{3}+(1-s) n_{4}+(-1+s) n_{5}-2 n_{6}>0 \\
-2 n_{1}+(-1+s) n_{2}+(1-s) n_{3}+2 n_{4}+(1+s) n_{5}+(-1-s) n_{6}>0 \\
-2 n_{1}+(-1-s) n_{2}+(1+s) n_{3}+2 n_{4}+(1-s) n_{5}+(-1+s) n_{6}>0 \\
(-1+s) n_{1}+(1-s) n_{2}+2 n_{3}+(1+s) n_{4}+(-1-s) n_{5}-2 n_{6}>0
\end{array}\right.
$$

- $20^{\text {th }}$ case $: B \rightarrow P, \quad P \rightarrow P$ et $A \rightarrow P$

$$
\left\{\begin{array}{l}
(1+s) n_{1}+(-1-s) n_{2}-2 n_{3}+(-1+s) n_{4}+(1-s) n_{5}+2 n_{6}>0 \\
(-1+s) n_{1}-2 n_{2}+(-1-s) n_{3}+(1+s) n_{4}+2 n_{5}+(1-s) n_{6}>0 \\
(1-s) n_{1}+(-1+s) n_{2}-2 n_{3}+(-1-s) n_{4}+(1+s) n_{5}+2 n_{6}>0 \\
(-1-s) n_{1}-2 n_{2}+(-1+s) n_{3}+(1-s) n_{4}+2 n_{5}+(1+s) n_{6}>0
\end{array}\right.
$$

- $27^{\text {th }}$ case $: B \rightarrow B, \quad P \rightarrow P$ et $A \rightarrow A$

$$
\left\{\begin{array}{l}
-2 n_{1}-n_{2}+n_{3}+2 n_{4}+n_{5}-n_{6}>0 \\
-n_{1}+n_{2}+2 n_{3}+n_{4}-n_{5}-2 n_{6}>0 \\
(1+s) n_{1}+(-1-s) n_{2}-2 n_{3}+(-1+s) n_{4}+(1-s) n_{5}+2 n_{6}>0 \\
2 n_{1}+(1+s) n_{2}+(-1-s) n_{3}-2 n_{4}+(-1+s) n_{5}+(1-s) n_{6}>0
\end{array}\right.
$$

\section{Appendix (III)}

- $\operatorname{Pr}($ case 4$)=\operatorname{Pr}(A \leftrightarrows B)-\operatorname{Pr}($ case 11$)$

- $\operatorname{Pr}($ case 7$)=\operatorname{Pr}(P \leftrightarrows B)-\operatorname{Pr}($ case 9$)$

- $\operatorname{Pr}($ case 8$)=\operatorname{Pr}(A \rightarrow P$ et $B \rightarrow P)-\operatorname{Pr}($ case 7$)-\operatorname{Pr}($ case 20)

- $\operatorname{Pr}($ case 12$)=\operatorname{Pr}(P \rightarrow B$ et $B \rightarrow A)-\operatorname{Pr}($ case 1$)$

- $\operatorname{Pr}($ case 15$)=\operatorname{Pr}(P \rightarrow A$ et $B \rightarrow B)-\operatorname{Pr}($ case 10$)$

- $\operatorname{Pr}($ case 16$)=\operatorname{Pr}(A \rightarrow P$ et $B \rightarrow B)-\operatorname{Pr}($ case 10$)$

- $\operatorname{Pr}($ case 19$)=\operatorname{Pr}(A \rightarrow B$ et $B \rightarrow P)-\operatorname{Pr}($ case 2)

- $\operatorname{Pr}($ case 21$)=\operatorname{Pr}(A \rightarrow A$ et $B \rightarrow B)-\operatorname{Pr}($ case 27$)$

- $\operatorname{Pr}($ case 23$)=\operatorname{Pr}(A \rightarrow A$ et $B \rightarrow A)-\operatorname{Pr}($ case 12$)-\operatorname{Pr}($ case 14$)$ 
- $\operatorname{Pr}($ case 24) $=\operatorname{Pr}(A \rightarrow A$ et $B \rightarrow P)-\operatorname{Pr}($ case 9$)$

- $\operatorname{Pr}($ case 25$)=\operatorname{Pr}(A \rightarrow B$ et $B \rightarrow B)-\operatorname{Pr}($ case 15$)-\operatorname{Pr}($ case 17$)$

\section{Appendix (IV)}

- $A \rightarrow P$ et $B \rightarrow A$

$$
\left\{\begin{array}{l}
n_{1}+2 n_{2}+n_{3}-n_{4}-2 n_{5}-n_{6}>0 \\
2 n_{1}+n_{2}-n_{3}-2 n_{4}-n_{5}+n_{6}>0 \\
(1-s) n_{1}+(-1+s) n_{2}-2 n_{3}+(-1-s) n_{4}+(1+s) n_{5}+2 n_{6}>0 \\
(-1-s) n_{1}-2 n_{2}+(-1+s) n_{3}+(1-s) n_{4}+2 n_{5}+(1+s) n_{6}>0
\end{array}\right.
$$

- $A \leftrightarrows B$

$$
\left\{\begin{array}{l}
n_{1}+2 n_{2}+n_{3}-n_{4}-2 n_{5}-n_{6}>0 \\
2 n_{1}+n_{2}-n_{3}-2 n_{4}-n_{5}+n_{6}>0 \\
-2 n_{1}+(-1-s) n_{2}+(1+s) n_{3}+2 n_{4}+(1-s) n_{5}+(-1+s) n_{6}>0 \\
(-1+s) n_{1}+(1-s) n_{2}+2 n_{3}+(1+s) n_{4}+(-1-s) n_{5}-2 n_{6}>0
\end{array}\right.
$$

- $P \leftrightarrows B$

$$
\left\{\begin{array}{l}
-n_{1}-2 n_{2}-n_{3}+n_{4}+2 n_{5}+n_{6}>0 \\
n_{1}-n_{2}-2 n_{3}-n_{4}+n_{5}+2 n_{6}>0 \\
(-1-s) n_{1}+(1+s) n_{2}+2 n_{3}+(1-s) n_{4}+(-1+s) n_{5}-2 n_{6}>0 \\
-2 n_{1}+(-1+s) n_{2}+(1-s) n_{3}+2 n_{4}+(1+s) n_{5}+(-1-s) n_{6}>0
\end{array}\right.
$$

- $A \rightarrow P$ et $B \rightarrow P$

$$
\left\{\begin{array}{l}
-n_{1}-2 n_{2}-n_{3}+n_{4}+2 n_{5}+n_{6}>0 \\
n_{1}-n_{2}-2 n_{3}-n_{4}+n_{5}+2 n_{6}>0 \\
(1-s) n_{1}+(-1+s) n_{2}-2 n_{3}+(-1-s) n_{4}+(1+s) n_{5}+2 n_{6}>0 \\
(-1-s) n_{1}-2 n_{2}+(-1+s) n_{3}+(1-s) n_{4}+2 n_{5}+(1+s) n_{6}>0
\end{array}\right.
$$

- $P \rightarrow B$ et $B \rightarrow A$

$$
\left\{\begin{array}{l}
n_{1}+2 n_{2}+n_{3}-n_{4}-2 n_{5}-n_{6}>0 \\
2 n_{1}+n_{2}-n_{3}-2 n_{4}-n_{5}+n_{6}>0 \\
(-1-s) n_{1}+(1+s) n_{2}+2 n_{3}+(1-s) n_{4}+(-1+s) n_{5}-2 n_{6}>0 \\
-2 n_{1}+(-1+s) n_{2}+(1-s) n_{3}+2 n_{4}+(1+s) n_{5}+(-1-s) n_{6}>0
\end{array}\right.
$$

- $P \rightarrow A$ et $B \rightarrow B$

$$
\left\{\begin{array}{l}
-2 n_{1}-n_{2}+n_{3}+2 n_{4}+n_{5}-n_{6}>0 \\
-n_{1}+n_{2}+2 n_{3}+n_{4}-n_{5}-2 n_{6}>0 \\
2 n_{1}+(1-s) n_{2}+(-1+s) n_{3}-2 n_{4}+(-1-s) n_{5}+(1+s) n_{6}>0 \\
(1-s) n_{1}+2 n_{2}+(1+s) n_{3}+(-1-s) n_{4}-2 n_{5}+(-1+s) n_{6}>0
\end{array}\right.
$$

- $A \rightarrow P$ et $B \rightarrow B$

$$
\left\{\begin{array}{l}
-2 n_{1}-n_{2}+n_{3}+2 n_{4}+n_{5}-n_{6}>0 \\
-n_{1}+n_{2}+2 n_{3}+n_{4}-n_{5}-2 n_{6}>0 \\
(1-s) n_{1}+(-1+s) n_{2}-2 n_{3}+(-1-s) n_{4}+(1+s) n_{5}+2 n_{6}>0 \\
(-1-s) n_{1}-2 n_{2}+(-1+s) n_{3}+(1-s) n_{4}+2 n_{5}+(1+s) n_{6}>0
\end{array}\right.
$$


- $A \rightarrow B$ et $B \rightarrow P$

$$
\left\{\begin{array}{l}
-n_{1}-2 n_{2}-n_{3}+n_{4}+2 n_{5}+n_{6}>0 \\
n_{1}-n_{2}-2 n_{3}-n_{4}+n_{5}+2 n_{6}>0 \\
-2 n_{1}+(-1-s) n_{2}+(1+s) n_{3}+2 n_{4}+(1-s) n_{5}+(-1+s) n_{6}>0 \\
(-1+s) n_{1}+(1-s) n_{2}+2 n_{3}+(1+s) n_{4}+(-1-s) n_{5}-2 n_{6}>0
\end{array}\right.
$$

- $A \rightarrow A$ et $B \rightarrow B$

$$
\left\{\begin{array}{l}
-2 n_{1}-n_{2}+n_{3}+2 n_{4}+n_{5}-n_{6}>0 \\
-n_{1}+n_{2}+2 n_{3}+n_{4}-n_{5}-2 n_{6}>0 \\
2 n_{1}+(1+s) n_{2}+(-1-s) n_{3}-2 n_{4}+(-1+s) n_{5}+(1-s) n_{6}>0 \\
(1+s) n_{1}+2 n_{2}+(1-s) n_{3}+(-1+s) n_{4}-2 n_{5}+(-1-s) n_{6}>0
\end{array}\right.
$$

- $A \rightarrow A$ et $B \rightarrow A$

$$
\left\{\begin{array}{l}
n_{1}+2 n_{2}+n_{3}-n_{4}-2 n_{5}-n_{6}>0 \\
2 n_{1}+n_{2}-n_{3}-2 n_{4}-n_{5}+n_{6}>0 \\
2 n_{1}+(1+s) n_{2}+(-1-s) n_{3}-2 n_{4}+(-1+s) n_{5}+(1-s) n_{6}>0 \\
(1+s) n_{1}+2 n_{2}+(1-s) n_{3}+(-1+s) n_{4}-2 n_{5}+(-1-s) n_{6}>0
\end{array}\right.
$$

- $A \rightarrow A$ et $B \rightarrow P$

$$
\left\{\begin{array}{l}
-n_{1}-2 n_{2}-n_{3}+n_{4}+2 n_{5}+n_{6}>0 \\
n_{1}-n_{2}-2 n_{3}-n_{4}+n_{5}+2 n_{6}>0 \\
2 n_{1}+(1+s) n_{2}+(-1-s) n_{3}-2 n_{4}+(-1+s) n_{5}+(1-s) n_{6}>0 \\
(1+s) n_{1}+2 n_{2}+(1-s) n_{3}+(-1+s) n_{4}-2 n_{5}+(-1-s) n_{6}>0
\end{array}\right.
$$

- $A \rightarrow B$ et $B \rightarrow B$

$$
\left\{\begin{array}{l}
-2 n_{1}-n_{2}+n_{3}+2 n_{4}+n_{5}-n_{6}>0 \\
-n_{1}+n_{2}+2 n_{3}+n_{4}-n_{5}-2 n_{6}>0 \\
-2 n_{1}+(-1-s) n_{2}+(1+s) n_{3}+2 n_{4}+(1-s) n_{5}+(-1+s) n_{6}>0 \\
(-1+s) n_{1}+(1-s) n_{2}+2 n_{3}+(1+s) n_{4}+(-1-s) n_{5}-2 n_{6}>0
\end{array}\right.
$$

\section{Appendix (IV)}

Our results depend on four different probabilities. The other calculations give us similar results. The case $\mathrm{N}^{\circ} 1$ has been presented previously, it's similar to the followings cases: 2, 9, 10, 11 and 27 . Here we present the three other probabilities :

- $14^{\text {th }}$ case $: B \rightarrow A, \quad P \rightarrow A$ and $A \rightarrow A$

This case is defined by the following system:

$$
\left\{\begin{array}{l}
2 n_{1}+(1-s) n_{2}+(-1+s) n_{3}-2 n_{4}+(-1-s) n_{5}+(1+s) n_{6}>0 \\
(1-s) n_{1}+2 n_{2}+(1+s) n_{3}+(-1-s) n_{4}-2 n_{5}+(-1+s) n_{6}>0 \\
2 n_{1}+(1+s) n_{2}+(-1-s) n_{3}-2 n_{4}+(-1+s) n_{5}+(1-s) n_{6}>0 \\
(1+s) n_{1}+2 n_{2}+(1-s) n_{3}+(-1+s) n_{4}-2 n_{5}+(-1-s) n_{6}>0
\end{array}\right.
$$

Let

$$
\left\{\begin{array}{l}
F_{1}: 2 n_{1}+(1-s) n_{2}+(-1+s) n_{3}-2 n_{4}+(-1-s) n_{5}+(1+s) n_{6}>0 \\
F_{2}:(1-s) n_{1}+2 n_{2}+(1+s) n_{3}+(-1-s) n_{4}-2 n_{5}+(-1+s) n_{6}>0 \\
F_{3}: 2 n_{1}+(1+s) n_{2}+(-1-s) n_{3}-2 n_{4}+(-1+s) n_{5}+(1-s) n_{6}>0 \\
F_{4}:(1+s) n_{1}+2 n_{2}+(1-s) n_{3}+(-1+s) n_{4}-2 n_{5}+(-1-s) n_{6}>0
\end{array}\right.
$$


and $\overrightarrow{v_{1}}, \overrightarrow{v_{2}}, \overrightarrow{v_{3}}, \overrightarrow{v_{4}}$ the normal vectors to the hyperplanes $F_{1}, F_{2}, F_{3}, F_{4}$ :

$$
\left\{\begin{array}{l}
\overrightarrow{v_{1}}=(2,1-s,-1+s,-2,-1-s, 1+s) \\
\overrightarrow{v_{2}}=(1-s, 2,1+s,-1-s,-2,-1+s) \\
\overrightarrow{v_{3}}=(2,1+s,-1-s,-2,-1+s, 1-s) \\
\overrightarrow{v_{4}}=(1+s, 2,1-s,-1+s,-2,-1-s)
\end{array}\right.
$$

We find the value of the 6 following angles $\alpha_{i j}$ :

$$
\begin{aligned}
& \alpha_{12}=\alpha_{34}=\frac{2 \pi}{3} \\
& \alpha_{13}=\alpha_{24}=\arccos \left(\frac{-3+s^{2}}{3+s^{2}}\right) \\
& \alpha_{14}=\alpha_{23}=\arccos \left(\frac{-3+s^{2}}{2\left(3+s^{2}\right)}\right)
\end{aligned}
$$

which implies that:

$$
\begin{aligned}
& d \alpha_{12}=d \alpha_{34}=0 \\
& d \alpha_{13}=d \alpha_{24}=\frac{-2 \sqrt{3}}{3+s^{2}} \\
& d \alpha_{14}=d \alpha_{23}=\frac{-4 s \sqrt{3}}{\left(3+s^{2}\right) \sqrt{9+10 s^{2}+s^{4}}}
\end{aligned}
$$

Then we can calculate the vertex $P_{123}=F_{1} \cap F_{2} \cap F_{3}$ by solving the system:

$$
P_{123}:\left\{\begin{array}{l}
2 n_{1}+(1-s) n_{2}+(-1+s) n_{3}-2 n_{4}+(-1-s) n_{5}+(1+s) n_{6}=0 \\
(1-s) n_{1}+2 n_{2}+(1+s) n_{3}+(-1-s) n_{4}-2 n_{5}+(-1+s) n_{6}=0 \\
2 n_{1}+(1+s) n_{2}+(-1-s) n_{3}-2 n_{4}+(-1+s) n_{5}+(1-s) n_{6}=0 \\
(1+s) n_{1}+2 n_{2}+(1-s) n_{3}+(-1+s) n_{4}-2 n_{5}+(-1-s) n_{6}>0
\end{array}\right.
$$

We derive the following results:

$$
P_{123}:\left\{\begin{array}{l}
n_{1}=-1 \\
n_{2}=\frac{s+1}{2} \\
n_{3}=\frac{s+1}{2} \\
n_{4}=-1 \\
n_{5}=\frac{-s+1}{2} \\
n_{6}=\frac{-s+1}{2}
\end{array}\right.
$$

By solving similar linear systems, we obtain the other vertices of the cone $C_{1}$ to be equal to:

$$
P_{124}:\left\{\begin{array}{l}
n_{1}=\frac{s+1}{2} \\
n_{2}=-1 \\
n_{3}=\frac{-s+1}{2} \\
n_{4}=\frac{-s+1}{2} \\
n_{5}=-1 \\
n_{6}=\frac{s+1}{2}
\end{array} \quad P_{134}:\left\{\begin{array}{l}
n_{1}=1 \\
n_{2}=\frac{s-1}{2} \\
n_{3}=\frac{s-1}{2} \\
n_{4}=1 \\
n_{5}=\frac{-s-1}{2} \\
n_{6}=\frac{-s-1}{2}
\end{array} \quad P_{234}:\left\{\begin{array}{l}
n_{1}=\frac{s-1}{2} \\
n_{2}=1 \\
n_{3}=\frac{-s-1}{2} \\
n_{4}=\frac{-s-1}{2} \\
n_{5}=1 \\
n_{6}=\frac{s-1}{2}
\end{array}\right.\right.\right.
$$


Also we compute:

$$
\begin{gathered}
\operatorname{vol}\left(F_{1}, F_{2}\right)=\operatorname{vol}\left(F_{3}, F_{4}\right)=\left(\widehat{P_{123}, P_{124}}\right)=\frac{2}{3} \pi \\
\operatorname{vol}\left(F_{1}, F_{3}\right)=\operatorname{vol}\left(F_{2}, F_{4}\right)=\left(\widehat{P_{123}, P_{134}}\right)=\arccos \left(\frac{-3+s^{2}}{3+s^{2}}\right) \\
\operatorname{vol}\left(F_{1}, F_{4}\right)=\operatorname{vol}\left(F_{2}, F_{3}\right)=\left(\widehat{P_{124}, P_{134}}\right)=\pi-\arccos \left(\frac{-3+s^{2}}{2\left(3+s^{2}\right)}\right)
\end{gathered}
$$

We find:

$$
\frac{d v o l(C)}{d s}=\frac{-4 \sqrt{3} \arccos \left(\frac{-3+s^{2}}{3+s^{2}}\right)}{3+s^{2}}-\frac{8 \sqrt{3} s\left(\pi-\arccos \left(\frac{-3+s^{2}}{2\left(3+s^{2}\right)}\right)\right)}{\left(3+s^{2}\right) \sqrt{9+10 s^{2}+s^{4}}}
$$

Finally we obtain:

$\operatorname{Pr}($ case $14, s)=\frac{1}{3}+\frac{1}{4 \pi^{2}} \int_{0}^{s}\left\{\frac{-4 \sqrt{3} \arccos \left(\frac{-3+t^{2}}{3+t^{2}}\right)}{3+t^{2}}-\frac{8 \sqrt{3} t\left(\pi-\arccos \left(\frac{-3+t^{2}}{2\left(3+t^{2}\right)}\right)\right)}{\left(3+t^{2}\right) \sqrt{9+10 t^{2}+t^{4}}}\right\} d t$

and $\operatorname{Pr}($ case 14$)=\operatorname{Pr}(s=1)=0.17821261$

The case 14 give us the similar probability than the case 17 and the case 20 .

- Subcase 1: $A \rightarrow P$ and $B \rightarrow A$

This subcase is defined by the following system:

$$
\left\{\begin{array}{l}
n_{1}+2 n_{2}+n_{3}-n_{4}-2 n_{5}-n_{6}>0 \\
2 n_{1}+n_{2}-n_{3}-2 n_{4}-n_{5}+n_{6}>0 \\
(1-s) n_{1}+(-1+s) n_{2}-2 n_{3}+(-1-s) n_{4}+(1+s) n_{5}+2 n_{6}>0 \\
(-1-s) n_{1}-2 n_{2}+(-1+s) n_{3}+(1-s) n_{4}+2 n_{5}+(1+s) n_{6}>0
\end{array}\right.
$$

Let

$$
\left\{\begin{array}{l}
F_{1}: n_{1}+2 n_{2}+n_{3}-n_{4}-2 n_{5}-n_{6}>0 \\
F_{2}: 2 n_{1}+n_{2}-n_{3}-2 n_{4}-n_{5}+n_{6}>0 \\
F_{3}:(1-s) n_{1}+(-1+s) n_{2}-2 n_{3}+(-1-s) n_{4}+(1+s) n_{5}+2 n_{6}>0 \\
F_{4}:(-1-s) n_{1}-2 n_{2}+(-1+s) n_{3}+(1-s) n_{4}+2 n_{5}+(1+s) n_{6}>0
\end{array}\right.
$$


and $\overrightarrow{v_{1}}, \overrightarrow{v_{2}}, \overrightarrow{v_{3}}, \overrightarrow{v_{4}}$ the normal vectors to the hyperplanes $F_{1}, F_{2}, F_{3}, F_{4}$ :

$$
\left\{\begin{array}{l}
\overrightarrow{v_{1}}=(1,2,1,-1,-2,-1) \\
\overrightarrow{v_{2}}=(2,1,-1,-2,-1,1) \\
\overrightarrow{v_{3}}=(1-s,-1+s,-2,-1-s, 1+s, 2) \\
\overrightarrow{v_{4}}=(-1-s,-2,-1+s, 1-s, 2,1+s)
\end{array}\right.
$$

We find the value of the 6 following angles $\alpha_{i j}$ :

$$
\begin{aligned}
& \alpha_{12}=\alpha_{34}=\frac{2 \pi}{3} \\
& \alpha_{13}=\alpha_{24}=\arccos \left(\frac{3}{2 \sqrt{9+3 s^{2}}}\right) \\
& \alpha_{14}=\arccos \left(\frac{3}{\sqrt{9+3 s^{2}}}\right) \\
& \alpha_{23}=\pi-\arccos \left(\frac{3}{2 \sqrt{9+3 s^{2}}}\right)
\end{aligned}
$$

which implies that:

$$
\begin{aligned}
d \alpha_{12} & =d \alpha_{34}=0 \\
d \alpha_{13} & =d \alpha_{24}=\frac{\sqrt{3} s}{\left(3+s^{2}\right) \sqrt{9+4 s^{2}}} \\
d \alpha_{14} & =\frac{\sqrt{3}}{3+s^{2}} \\
d \alpha_{23} & =\frac{-\sqrt{3} s}{\left(3+s^{2}\right) \sqrt{9+4 s^{2}}}
\end{aligned}
$$

Then we can calculate the vertex $P_{123}=F_{1} \cap F_{2} \cap F_{3}$ by solving the system:

$$
P_{123}:\left\{\begin{array}{l}
n_{1}+2 n_{2}+n_{3}-n_{4}-2 n_{5}-n_{6}=0 \\
2 n_{1}+n_{2}-n_{3}-2 n_{4}-n_{5}+n_{6}=0 \\
(1-s) n_{1}+(-1+s) n_{2}-2 n_{3}+(-1-s) n_{4}+(1+s) n_{5}+2 n_{6}=0 \\
(-1-s) n_{1}-2 n_{2}+(-1+s) n_{3}+(1-s) n_{4}+2 n_{5}+(1+s) n_{6}>0
\end{array}\right.
$$

We derive the following results:

$$
P_{123}:\left\{\begin{array}{l}
n_{1}=1 \\
n_{2}=1 \\
n_{3}=-2 \\
n_{4}=1 \\
n_{5}=1 \\
n_{6}=-2
\end{array}\right.
$$


By solving similar linear systems, we obtain the other vertices of the cone $C_{1}$ to be equal to:

$$
P_{124}:\left\{\begin{array}{l}
n_{1}=1 \\
n_{2}=-2 \\
n_{3}=1 \\
n_{4}=1 \\
n_{5}=-2 \\
n_{6}=1
\end{array} \quad P_{134}:\left\{\begin{array}{l}
n_{1}=-1+s \\
n_{2}=2 \\
n_{3}=-1-s \\
n_{4}=-1-s \\
n_{5}=2 \\
n_{6}=-1+s
\end{array} \quad P_{234}:\left\{\begin{array}{l}
n_{1}=2 \\
n_{2}=-1+s \\
n_{3}=-1+s \\
n_{4}=2 \\
n_{5}=-1-s \\
n_{6}=-1-s
\end{array}\right.\right.\right.
$$

Also we compute:

$$
\begin{gathered}
\operatorname{vol}\left(F_{1}, F_{2}\right)=\operatorname{vol}\left(F_{3}, F_{4}\right)=\left(\widehat{P_{123}, P_{124}}\right)=\frac{2}{3} \pi \\
\operatorname{vol}\left(F_{1}, F_{3}\right)=\operatorname{vol}\left(F_{2}, F_{4}\right)=\operatorname{vol}\left(F_{2}, F_{3}\right)=\left(\widehat{P_{123, P_{134}}}\right)=\arccos \left(\frac{3}{2 \sqrt{9+3 s^{2}}}\right) \\
\operatorname{vol}\left(F_{1}, F_{4}\right)=\left(\widehat{P_{124}, P_{134}}\right)=\pi-\arccos \left(\frac{3}{\sqrt{9+3 s^{2}}}\right)
\end{gathered}
$$

We find:

$$
\frac{d v o l(C)}{d s}=\frac{s \sqrt{3} \arccos \left(\frac{3}{2 \sqrt{9+3 s^{2}}}\right)}{\left(3+s^{2}\right) \sqrt{9+4 s^{2}}}+\frac{\sqrt{3}\left\{\pi-\arccos \left(\frac{3}{\sqrt{9+3 s^{2}}}\right)\right\}}{3+s^{2}}
$$

Finally we obtain:

$\operatorname{Pr}($ Subcase $1, s)=\frac{1}{4 \pi^{2}} \int_{0}^{s}\left\{\frac{t \sqrt{3} \arccos \left(\frac{3}{2 \sqrt{9+3 t^{2}}}\right)}{\left(3+t^{2}\right) \sqrt{9+4 t^{2}}}+\frac{\sqrt{3}\left\{\pi-\arccos \left(\frac{3}{\sqrt{9+3 t^{2}}}\right)\right\}}{3+t^{2}}\right\} d t$

and $\operatorname{Pr}($ Subcase 1$)=\operatorname{Pr}(s=1)=0.04027691$

The case $(A \rightarrow P$ and $B \rightarrow A)$ give us the similar probability than the followings cases:

$$
\begin{array}{rl}
A \leftrightarrows B & A \rightarrow P \text { and } B \rightarrow B) \\
P \leftrightarrows B & A \rightarrow B \text { and } B \rightarrow P \\
P \rightarrow B \text { and } B \rightarrow A & A \rightarrow A \text { and } B \rightarrow B \\
P \rightarrow A \text { and } B \rightarrow B & A \rightarrow A \text { and } B \rightarrow P
\end{array}
$$


- Subcase 2: $A \rightarrow P$ and $B \rightarrow P$

This subcase is defined by the following system:

$$
\left\{\begin{array}{l}
-n_{1}-2 n_{2}-n_{3}+n_{4}+2 n_{5}+n_{6}>0 \\
n_{1}-n_{2}-2 n_{3}-n_{4}+n_{5}+2 n_{6}>0 \\
(1-s) n_{1}+(-1+s) n_{2}-2 n_{3}+(-1-s) n_{4}+(1+s) n_{5}+2 n_{6}>0 \\
(-1-s) n_{1}-2 n_{2}+(-1+s) n_{3}+(1-s) n_{4}+2 n_{5}+(1+s) n_{6}>0
\end{array}\right.
$$

Let

$$
\left\{\begin{array}{l}
F_{1}:-n_{1}-2 n_{2}-n_{3}+n_{4}+2 n_{5}+n_{6}>0 \\
F_{2}: n_{1}-n_{2}-2 n_{3}-n_{4}+n_{5}+2 n_{6}>0 \\
F_{3}:(1-s) n_{1}+(-1+s) n_{2}-2 n_{3}+(-1-s) n_{4}+(1+s) n_{5}+2 n_{6}>0 \\
F_{4}:(-1-s) n_{1}-2 n_{2}+(-1+s) n_{3}+(1-s) n_{4}+2 n_{5}+(1+s) n_{6}>0
\end{array}\right.
$$

and $\overrightarrow{v_{1}}, \overrightarrow{v_{2}}, \overrightarrow{v_{3}}, \overrightarrow{v_{4}}$ the normal vectors to the hyperplanes $F_{1}, F_{2}, F_{3}, F_{4}$ :

$$
\left\{\begin{array}{l}
\overrightarrow{v_{1}}=(-1,-2,-1,1,2,1) \\
\overrightarrow{v_{2}}=(1,-1,-2,-1,1,2) \\
\overrightarrow{v_{3}}=(1-s,-1+s,-2,-1-s, 1+s, 2) \\
\overrightarrow{v_{4}}=(-1-s,-2,-1+s, 1-s, 2,1+s)
\end{array}\right.
$$

We find the value of the 6 following angles $\alpha_{i j}$ :

$$
\begin{aligned}
& \alpha_{12}=\alpha_{34}=\frac{2 \pi}{3} \\
& \alpha_{13}=\alpha_{24}=\pi-\arccos \left(\frac{3}{2 \sqrt{9+3 s^{2}}}\right) \\
& \alpha_{14}=\alpha_{23}=\pi-\arccos \left(\frac{3}{\sqrt{9+3 s^{2}}}\right)
\end{aligned}
$$

which implies that:

$$
\begin{aligned}
& d \alpha_{12}=d \alpha_{34}=0 \\
& d \alpha_{13}=d \alpha_{24}=\frac{-\sqrt{3} s}{\left(3+s^{2}\right) \sqrt{9+4 s^{2}}} \\
& d \alpha_{14}=d \alpha_{23}=\frac{-\sqrt{3}}{3+s^{2}}
\end{aligned}
$$

Then we can calculate the vertex $P_{123}=F_{1} \cap F_{2} \cap F_{3}$ by solving the system:

$$
P_{123}:\left\{\begin{array}{l}
-n_{1}-2 n_{2}-n_{3}+n_{4}+2 n_{5}+n_{6}=0 \\
n_{1}-n_{2}-2 n_{3}-n_{4}+n_{5}+2 n_{6}=0 \\
(1-s) n_{1}+(-1+s) n_{2}-2 n_{3}+(-1-s) n_{4}+(1+s) n_{5}+2 n_{6}=0 \\
(-1-s) n_{1}-2 n_{2}+(-1+s) n_{3}+(1-s) n_{4}+2 n_{5}+(1+s) n_{6}>0
\end{array}\right.
$$


We derive the following results:

$$
P_{123}:\left\{\begin{array}{l}
n_{1}=1 \\
n_{2}=1 \\
n_{3}=-2 \\
n_{4}=1 \\
n_{5}=1 \\
n_{6}=-2
\end{array}\right.
$$

By solving similar linear systems, we obtain the other vertices of the cone $C_{1}$ to be equal to:

$$
P_{124}:\left\{\begin{array}{l}
n_{1}=1 \\
n_{2}=-2 \\
n_{3}=1 \\
n_{4}=1 \\
n_{5}=-2 \\
n_{6}=1
\end{array} \quad P_{134}:\left\{\begin{array}{l}
n_{1}=-1+s \\
n_{2}=2 \\
n_{3}=-1-s \\
n_{4}=-1-s \\
n_{5}=2 \\
n_{6}=-1+s
\end{array} \quad P_{234}:\left\{\begin{array}{l}
n_{1}=-1-s \\
n_{2}=-1-s \\
n_{3}=2 \\
n_{4}=-1+s \\
n_{5}=-1+s \\
n_{6}=2
\end{array}\right.\right.\right.
$$

Also we compute:

$$
\begin{gathered}
\operatorname{vol}\left(F_{1}, F_{2}\right)=\operatorname{vol}\left(F_{3}, F_{4}\right)=\left(\widehat{P_{123}, P_{124}}\right)=\frac{2}{3} \pi \\
\operatorname{vol}\left(F_{1}, F_{3}\right)=\operatorname{vol}\left(F_{2}, F_{4}\right)=\left(\widehat{P_{123}, P_{134}}\right)=\arccos \left(\frac{3}{2 \sqrt{9+3 s^{2}}}\right) \\
\operatorname{vol}\left(F_{1}, F_{4}\right)=\operatorname{vol}\left(F_{2}, F_{3}\right)=\left(\widehat{P_{124}, P_{134}}\right)=\pi-\arccos \left(\frac{3}{\sqrt{9+3 s^{2}}}\right)
\end{gathered}
$$

We find:

$$
\frac{d v o l(C)}{d s}=\frac{-2 s \sqrt{3} \arccos \left(\frac{3}{2 \sqrt{9+3 s^{2}}}\right)}{\left(3+s^{2}\right) \sqrt{9+4 s^{2}}}-\frac{2 \sqrt{3}\left\{\pi-\arccos \left(\frac{3}{\sqrt{9+3 s^{2}}}\right)\right\}}{3+s^{2}}
$$

Finally we obtain:

$\operatorname{Pr}($ Subcase $2, s)=\frac{1}{3}+\frac{1}{4 \pi^{2}} \int_{0}^{s}\left\{\frac{-2 t \sqrt{3} \arccos \left(\frac{3}{2 \sqrt{9+3 t^{2}}}\right)}{\left(3+t^{2}\right) \sqrt{9+4 t^{2}}}-\frac{2 \sqrt{3}\left\{\pi-\arccos \left(\frac{3}{\sqrt{9+3 t^{2}}}\right)\right\}}{3+t^{2}}\right\} d t(94)$ and $\operatorname{Pr}($ Subcase 2$)=\operatorname{Pr}(s=1)=0.25277950$ This case $(A \rightarrow P$ and $B \rightarrow P)$ give the similar probability than the followings cases:

$$
A \rightarrow A \text { and } B \rightarrow A \quad A \rightarrow B \text { and } B \rightarrow B
$$




\section{References}

Barberà, S. and Beviá, S.: (2002), Self-selection consistent functions, Journal of Economic Theory 105, 263-277.

Barberà, S. and Jackson, M.O.: (2004), Choosing how to choose: Self-stable majority rules and constitutions, The Quarterly Journal of Economics 119(3), 10111048.

Berg, S. and Lepelley, D.: (1994), On probability models in voting theory, Statistica Neerlandica 48, 133-146.

Gehrlein, W.: (2002), Condorcet's paradox and the likelihood of its occurrence: different perspectives on balanced preferences, Theory and decision 52, 171-199.

Gehrlein, W.V.: (2006), Condorcet's Paradox, Theory and Decision Library C 40, Hardcover.

Gehrlein, W.V. and Valognes, F.: (2001), Condorcet efficiency: A preference for indifference, Social Choice and Welfare 18(1), 193-205.

Guilbauld, G.T.: (1952), Les théories de l'intérêt général et le problème logique de l'agrégation, Économie Appliquée 5, 50-584.

Houy, N.: (2005), De la stabilité des procédures et constitutions en théorie du choix social, PhD Thesis, University of Paris I.

Kellerhals, R.: (1989), On the volume of hyperbolic polyhedra, Mathematische Annalen 285, 541-569.

Koray, S.: (2000), Self-selective social choice functions verify arrow and gibbardsatterthwaite theorems, Econometrica 68(4), 981-995.

Koray, S. and Unel, B.: (2003), Charactrization of self-selective social choice functions on the tops-only domain, Social Choice and Welfare 20, 495-507.

Marchant, T.: (2001), The probability of ties with scoring methods: Some results, Social Choice and Welfare 18(4), 709-735.

May, K.: (1952), A Set of Independent, Necessary and Sufficient Conditions for Simple Majority Decision. Econometrica 20:680-684.

Merlin, V.: (1996), L'agrégation des préférences individuelles: les règles positionelles itératives et la méthode de Copeland, PhD thesis, University of Caen Law-Normandy.

Merlin, V. and Valognes, F.: (2004), The impact of indifferent voters on the likelihood of some voting paradoxes, Mathematical Social Sciences 48, 343-361.

Merlin, V., Tataru, M. and Valognes, F.: (2000), On the probability that all decision rules select the same winner, Journal Of mathamatical Economics 33, 183-207. Merlin, V., Tataru, M. and Valognes, F.: (2002), On the likelihood of condorcet's profiles, Social Choice and Welfare 19, 193-206. 
Merlin, V. and Tataru, M.: (1997), On the relationship of the condorcet winner and positional voting rules, Mathematical Social Sciences 34, 81-90.

Milnor, J.: (1982), Hyperbolic Geometry: the first 150 years, Bull AMS 6, 9-24.

Saari, D.: (1994), Geometry of voting. Springer-Verlag, Berlin.

Saari, D.: (1995), Basic Geometry of voting, New York, Springer.

Saari, D. and Tataru, M.: (1999), The likelihood of dubious election outcomes, Economic Theory 13, 345-363. 